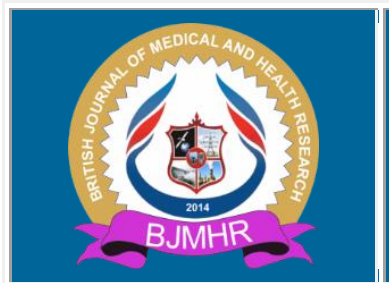

\title{
BJMHR
}

British Journal of Medical and Health Research

Journal home page: www.bjmhr.com

\section{CAD/CAM Milling versus Rapid Prototyping Surgical Guide Techniques in Dental Implant Placement}

\section{Marwa Ezzat Sabet ${ }^{1}$, Fardos Nabil Rizk², Hebatallah Tarek Mohammed ${ }^{3}$, Adham} \\ Omar Osman Elghamry, 5* $^{*}$ \\ 1. Ain Shams University/ Faculty of Dentistry/ Professor and Chairman of Prosthodontics \\ Department, Egypt. \\ 2. British University in Egypt/ Faculty of Dentistry/ Professor and Chairman of Prosthodontics \\ Department, Egypt. \\ 3. Ain Shams University/ Faculty of Dentistry/ Associate Professor of Removable \\ Prosthodontics, Egypt. \\ 4. Cairo University/ Faculty of Dentistry/B.D.S. \\ 5. British University in Egypt/ Faculty of Dentistry/ Demonstrator of Removable \\ Prosthodontics Department, Egypt.
}

\section{ABSTRACT}

This study was done to compare between the accuracy of surgical guides in implant placement using different techniques of construction; CAD/CAM milling and Rapid Prototyping (3D printing). 28 implants divided equally into two groups, group I implant inserted using CAD/CAM milling surgical guides while group II implant inserted using 3D printed (Rapid Prototyping) surgical guides. A pre-operative CBCT was taken to determine the virtual implant location regarding Coronal, apical and angular positions, after implant placement, a postoperative CBCT was taken and the Blue sky plan computer software was used to match the pre and postoperative CBCT images, to compare Angular, coronal, and apical deviation of the virtual and the actual implants positions by Superimposition with the post-operative CBCT. There were a statistically significant higher coronal, apical and angular deviation mean values between the virtual and the actual implants placement of group II than of group I. The CAD/CAM milled surgical guides had superior results than 3D printing surgical guides.

Keywords: CAD/CAM, 3Dprinting, surgical guides. 


\section{INTRODUCTION}

Osseo-integrated implants are a practical substitute to the conventional prosthodontics; nevertheless, designing a prosthesis which is implant supported with proper function and esthetics is a challenge. Precise accuracy in planning and conduction of surgical steps is vital for assuring a high-success probability exclusive of iatrogenic damage. The success of implant placement primarily relies on well-organized treatment planning and correctly performed surgery. Disorderly placed implant is a very common problem that regularly complicates not only the clinical, but also the laboratory procedures of superstructures. This actually dictates a close teamwork between prosthodontists and surgeons to work conjointly as a single unit that will smoothen the accurate construction of the surgical stent or surgical guide.

A surgical stent is an appliance utilized for radiographic assessment of the available bone regarding height and width pre-operatively or intra-operatively to provide the ideal site for implant placements ${ }^{1}$. Surgical templates not only aid in diagnosis and treatment planning, but also eases proper positioning and correct angulation of the implant body in the bone. Furthermore, restoration- driven implant placement accomplished with a surgical guide template, for sure, decreases the clinical and laboratory complications. Thus, the increasing demand for dental implant placement using surgical guides has resulted in more advanced techniques for the fabrication of these templates. ${ }^{2,3}$

Guides should be constructed of transparent material, stable and firm when in position. It should cover sufficient teeth to stabilize its location, and when teeth are absent, they should extend onto the un-reflected soft tissue regions ${ }^{3}$. A surgical guide is supported by the teeth, mucosa or bone and is usually made of polymer. It has pre-drilled holes and, during the dental implant surgery, the surgeon uses these holes to guide the osteotomy at the anticipated locations and angulations in the patient's subsequent implantation site ${ }^{4}$. Several computer guided surgical stents fabrication methods have been advocated over the past several years including designrelated processing and milling based on coordinate synchronization. In design-related processing, a template is designed on a computer which is then used to construct a surgical stent either by subtractive or additive method. Then this study was prompted to evaluate which 3-D surgical stent is more accurate in implant placement.

\section{MATERIALS AND METHOD}

Mandibular bounded partially edentulous patients were selected from the out-patient clinic of Prosthodontics Department, Faculty of Dentistry, Ain Shams University with the following Inclusion Criteria: (1) Age ranging from 45-65 years.(2) Angle class I maxilla-mandibular relationship and sufficient inter-arch spaces.(3) Presence of sufficient bone height (14mm) and width $(6 \mathrm{~mm})$ for the placement of implant fixtures without using regenerative techniques.(4) 
Residual alveolar ridge covered with healthy mucosa of even thickness and free of any inflammation. While the exclusion criteria was; (1) Uncontrolled systemic diseases that contraindicate the surgical procedures, complicate the procedure or delay the healing period time. (2) systemic diseases affecting bone metabolism.(3) Renal diseases or diuretics consumption. (4) Bone diseases and abnormal and para-functional habits as (bruxism, clenching, tongue thrusting). (5) Heavy smoking, Drug or alcohol abuse. (6) Chemotherapy or Radiotherapy or any disorders to the implant area related to a history of radiation therapy to the head and neck, neoplasia, or bone augmentation to the implant site.

\section{Patients' Grouping:}

28 implants, 14 in each group were inserted into selected patients randomly divided into two groups:

Group I: 14 implants inserted in bounded partially edentulous mandibular arches using CAD/CAM milling surgical guides. While

Group II: 14 implants inserted in bounded partially edentulous mandibular arches using completely limiting 3D printed (Rapid Prototyping) surgical guides. After an informed consent document had been signed by the patient, treatment plan was completed, primary maxillary and mandibular impressions were made using alginate (Cavex - Holland.) impression material in properly selected stock trays after their modification. CBCT image was obtained in Digital Imaging and Communications in Medicine (DICOM) format, a pre-operative CBCT was taken to evaluate and measure the available bone width and height and to determine the virtual implant location regarding coronal and apical and angular positions. (Fig. 1). With Blue Sky Plan (Blue sky program version 3.40.6 software format)( Blue sky Bio, LLC, Grayslake USA.), implants were virtually placed according to the bone anatomy, bone height and width (buccolingual and mesiodistal) and prosthetic design. (Figure 2) 


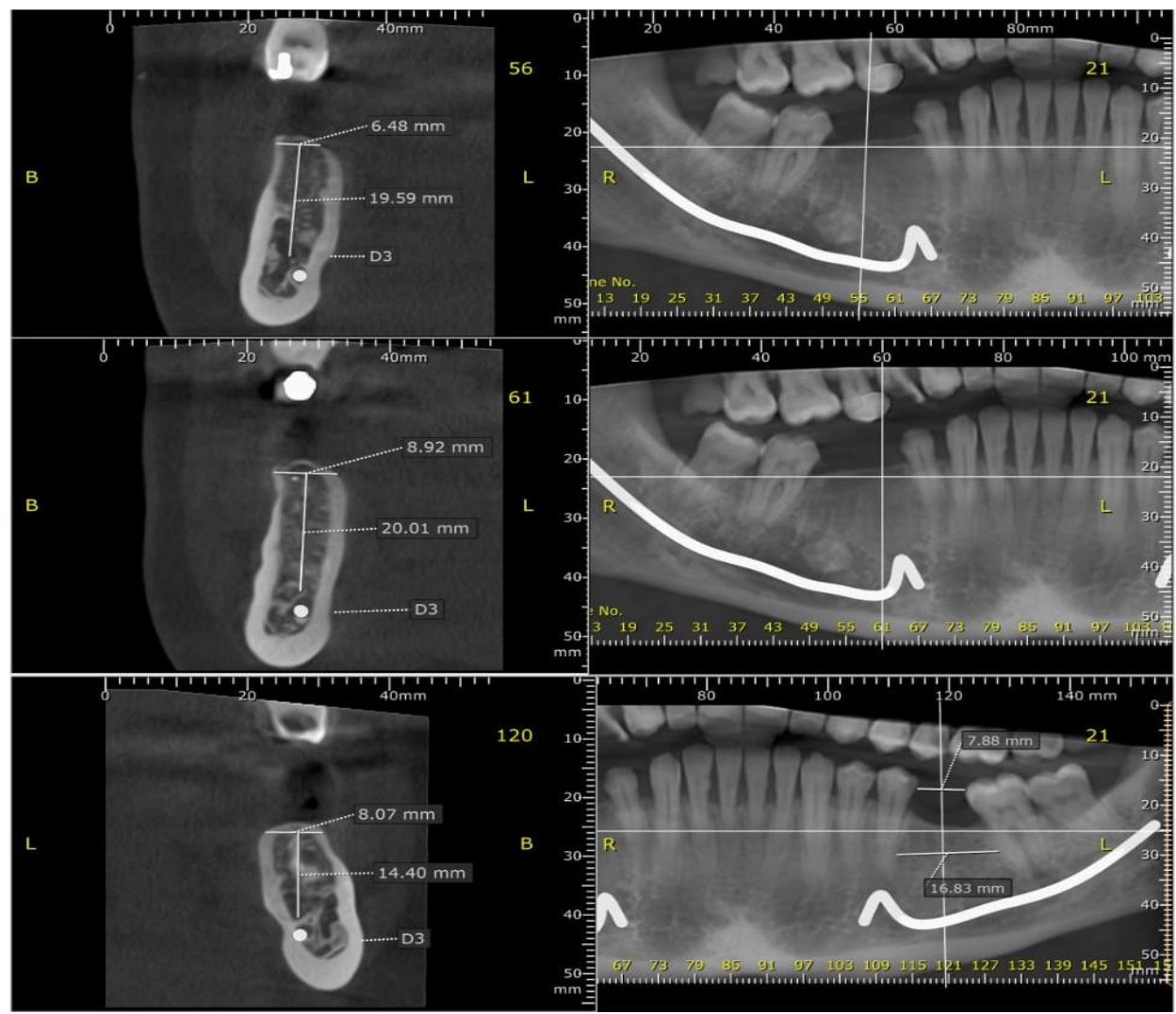

Figure 1: Pre-operative CBCT with bone height and width measurements

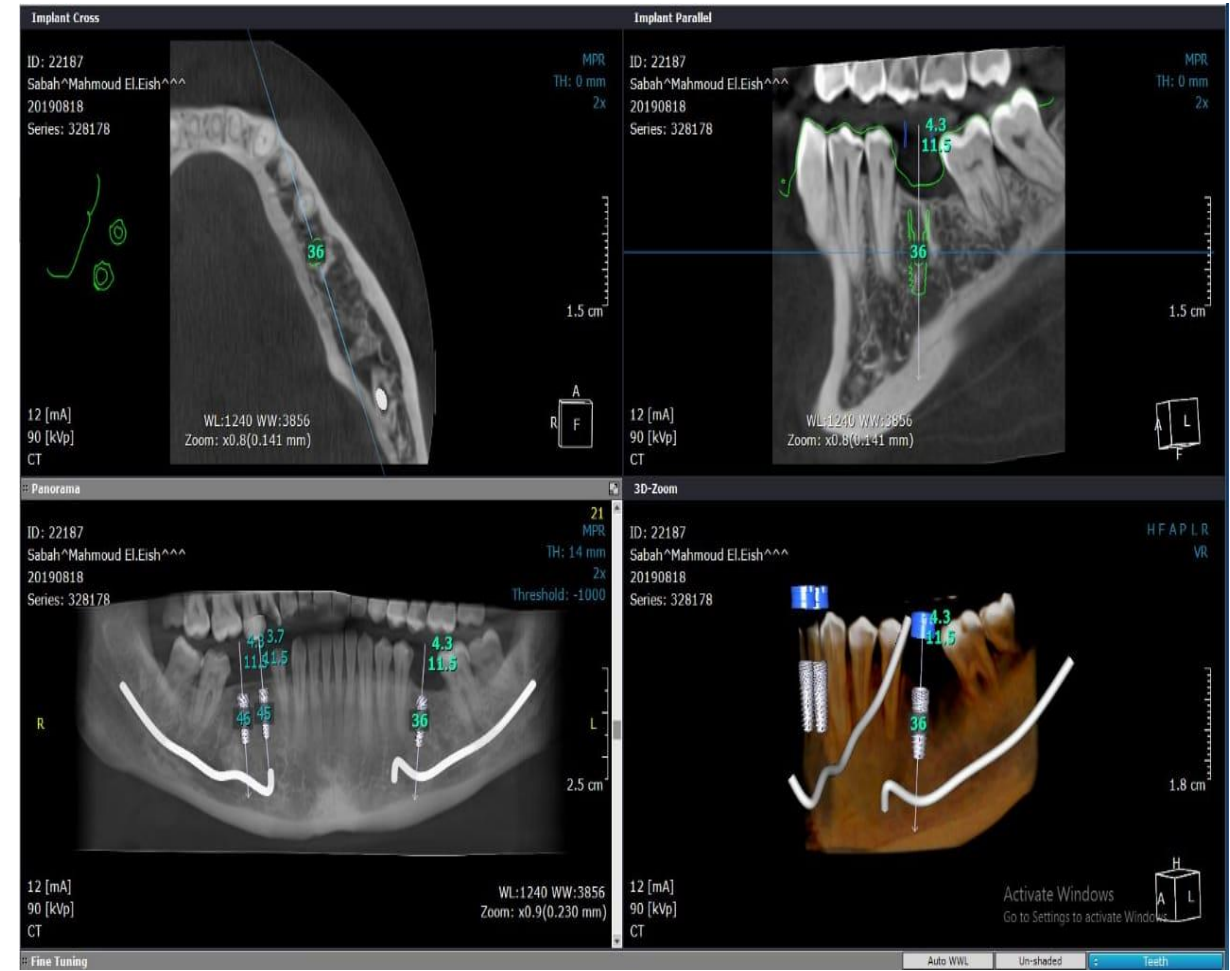

Figure 2: Virtual implant planning.

Study casts were scanned using optical scanner (Shera eco-scan 7 scanner.) to produce a virtual 3D model on which the surgical guides were constructed. Once the computer planning is accomplished, this plan is saved as a ".STL" file format and either uploaded into a 3D printer machine (Rapid prototyping machine P380, EOS, Munich, Germany), or to CAD/CAM machine (CNC milling machine. VHF -mill K5, five axis, Germany). Figure 3, 4. 


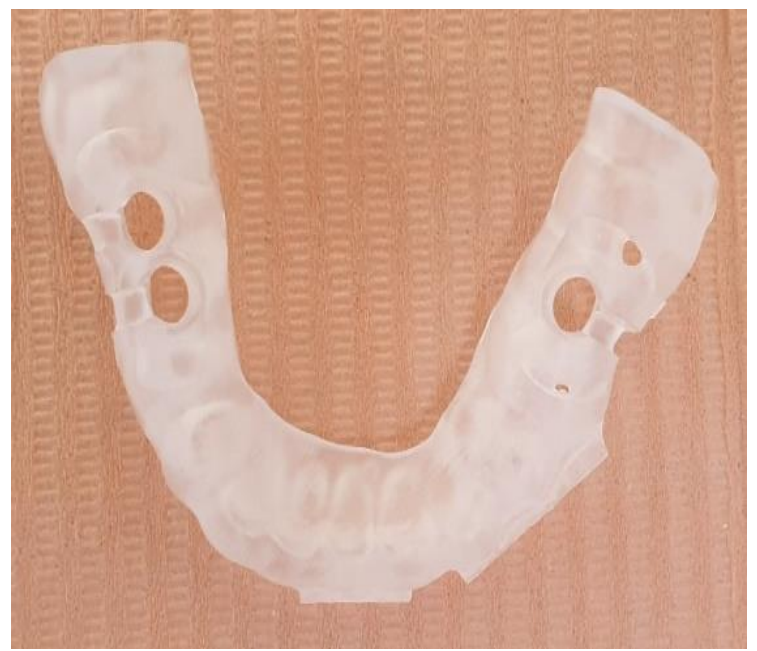

Figure 3: CAD/CAM guide.

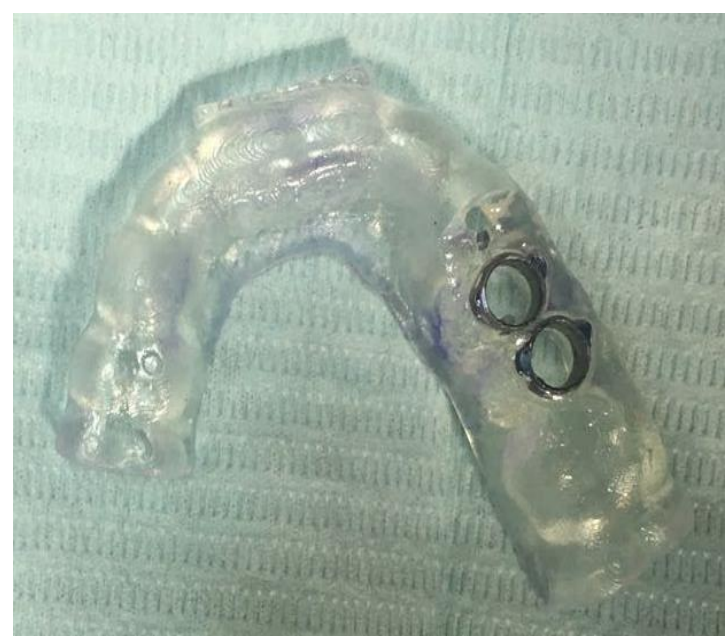

Figure 4: 3D printing guide.

\section{Surgical procedures and implant placement:}

Pre-medications include antibiotic (Clavulanate-potentiated Amoxicillin) 1gm/12 hours the day before surgery and continued for five days after, anti-inflammatory (Diclofenac sodium) $50 \mathrm{mg}$ and mouth wash (Chlorhexidine) were prescribed 3 times daily prior to surgery. After checking the local anaesthesia; the surgical stent was disinfected and inserted into the patient's mouth and supported by the remaining teeth. Osteotomy was performed till reaching the final drill of the simple guide with $2.8 \mathrm{~mm}$ diameter and $11.5 \mathrm{~mm}$ length. After stent removal, the implant (Neobiotech j dental (Neobiotech Co., Ltd. Guro-gu, Seoul, Korea) with diameter 4 $\mathrm{mm}$ and length $11.5 \mathrm{~mm}$ ) was inserted through the osteotomy manually then continued using a ratchet.

\section{Post- operative care:}

Cold soft diet was recommended. Antibiotic, analgesic and anti-inflammatory that were prescribed to the patient before the surgery, continued for the following five days. The patient was instructed to come back the next day to check.

\section{Post- Operative Imaging \& Image Super-Imposition:}


Patients were recalled three days after implant insertion for another CBCT scan. This Postoperative CBCT was performed using the same pre-operative CBCT parameters and the same machine. The Blue sky plan computer software was used to match the pre and postoperative CBCT images, to compare Angular deviation, coronal deviation, and apical deviation of the virtual and the actual implants positions by superimposition with the postoperative CBCT after the implant insertion. Evaluation of Accuracy: The difference between the planned and the actual implants in three-dimensional view was calculated in two parts, the coronal and the apical parts, and is called the total sum of the coronal and apical differences. In addition, the angular deviation which is the three-dimensional angle between the long axis of the planned and the actual implant was calculated, tabulated, and statistically analyzed. Figure 5

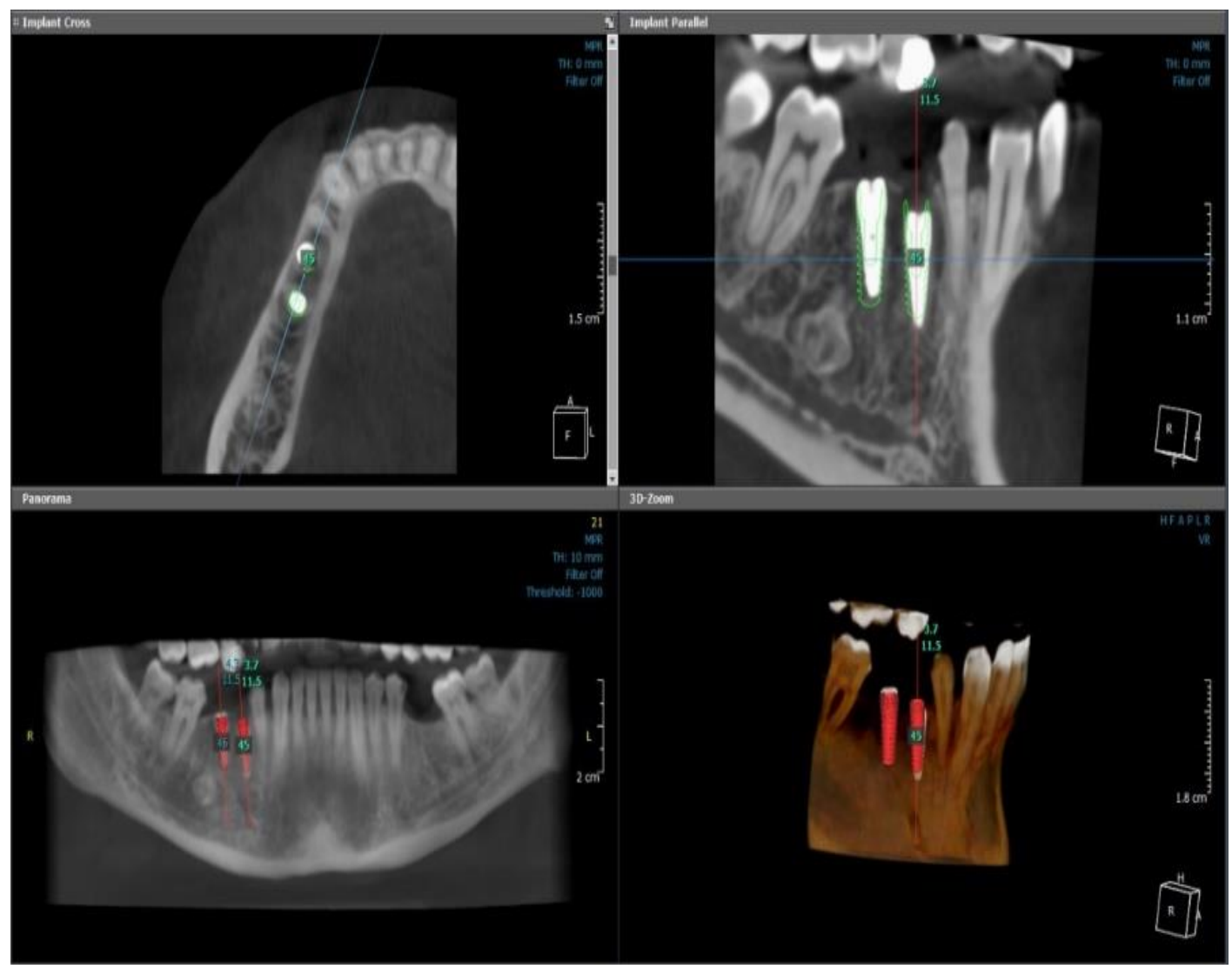

Figure 5: Superimposition of virtual and post-operative CBCT.

\section{RESULTS AND DISCUSSION}

Statistical analysis was performed using IBM SPSS Statistics Version 2.1 for Windows. Data was presented as mean and standard deviation (SD). The significance level was set at $\mathrm{P} \leq 0.05$. Kolmogorov-Smirnov and Shapiro-Wilk tests were used to assess data normality.

Since all data showed normal distribution, Iindependent Student t-test ( 2 independent samples) was performed to compare angular, coronal and apical deviation between $3 \mathrm{D}$ printing and CAD/CAM surgical guides.

Comparison of angular deviation between 3D printing and CAD/CAM surgical guides: 
Table 1: Mean, Standard deviation and P-value for the effect of surgical guide technique on angular deviation (degrees).

*: Significant at $P \leq 0.05$

\begin{tabular}{lll}
\hline & \multicolumn{2}{l}{ Angular Deviation } \\
& Milling & 3D Printing \\
\hline Mean & 0.424 & 4.760 \\
Standard Deviation & 0.098 & 0.957 \\
P-Value & $<0.0001^{*}$ \\
\hline
\end{tabular}

Independent student t-test (Table 1) showed that 3D printing (group II) had a statistically significant higher angular deviation than milling (group I) surgical guides $(\mathrm{P}<0.0001)$.

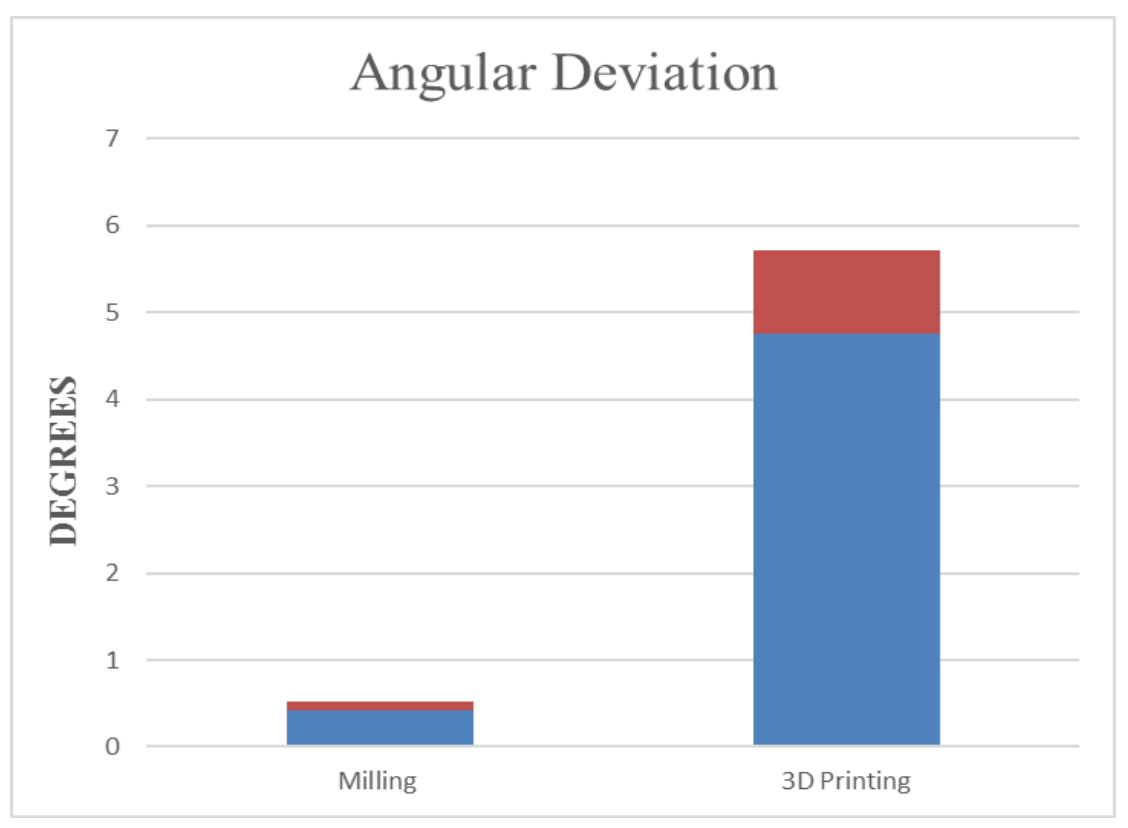

Figure 6: Bar chart representing mean and standard deviation values for angular deviation in milling and 3D printing surgical guides.

Comparison of coronal deviation between 3D printing and CAD/CAM surgical guides:

Table 2: Mean, Standard deviation and P-value for the effect of surgical guide technique on coronal deviation $(\mu \mathrm{m})$.

NS: non-significant at $P \leq 0.05$

\begin{tabular}{lll}
\hline & \multicolumn{2}{l}{ Coronal Deviation } \\
& Milling & 3D Printing \\
\hline Mean & 0.342 & 0.542 \\
Standard Deviation & 0.098 & 0.146 \\
P-Value & $0.039^{*}$ & \\
\hline
\end{tabular}

Independent student t-test (Table 2) showed that 3D printing (group II) had a statistically significant higher coronal deviation than milling (group I) surgical guides $(\mathrm{P}=0.039)$. 


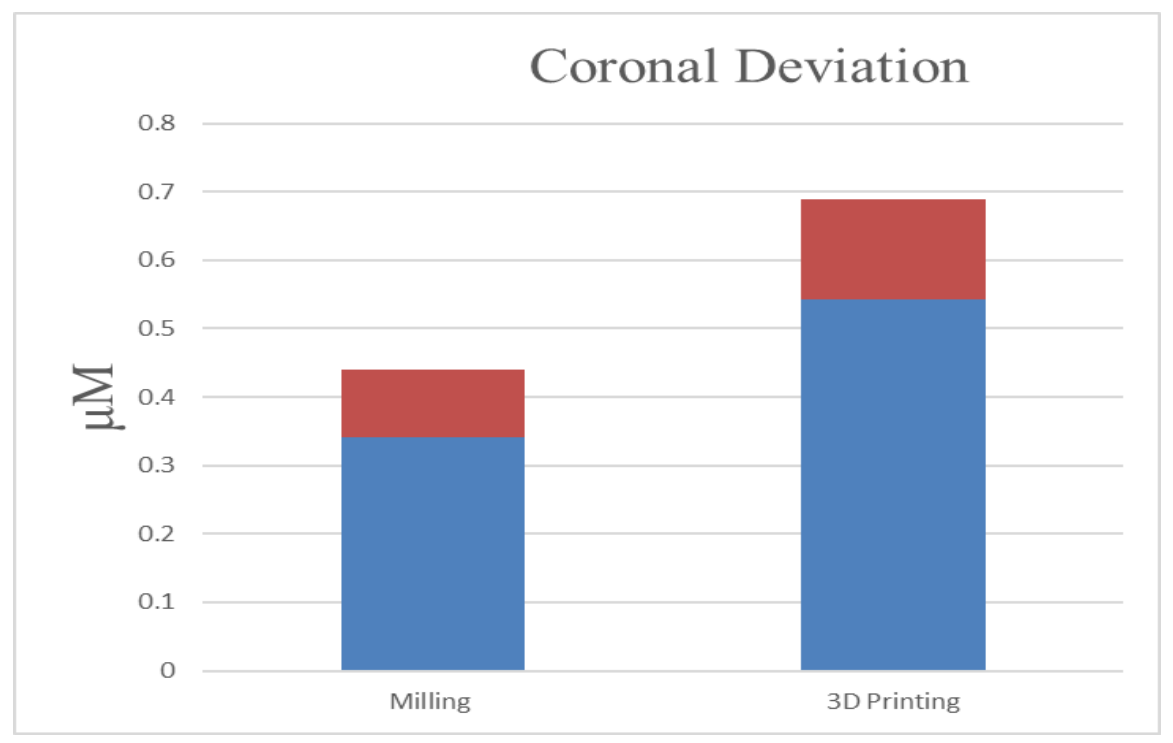

Figure 7: Bar chart representing mean and standard deviation values for coronal deviation in milling and 3D printing surgical guides.

Comparison of apical deviation between 3D printing and CAD/CAM surgical guides:

Table 3: Mean, Standard deviation and P-value for the effect of surgical guide technique on apical deviation $(\mu \mathrm{m})$.

\begin{tabular}{lll}
\hline & \multicolumn{2}{l}{ Apical Deviation } \\
& Milling & 3D Printing \\
\hline Mean & 0.384 & 1.128 \\
Standard Deviation & 0.050 & 0.252 \\
P-Value & $0.002 *$ & \\
\hline
\end{tabular}

NS: non-significant at $\frac{\mathrm{P}-\mathrm{V} \text { alue }}{P \leq 0.05}$

Independent student t-test (Table 3) showed that 3D printing (group II) showed a statistically significant higher apical deviation than milling (group I) surgical guides $(\mathrm{P}=0.002)$.

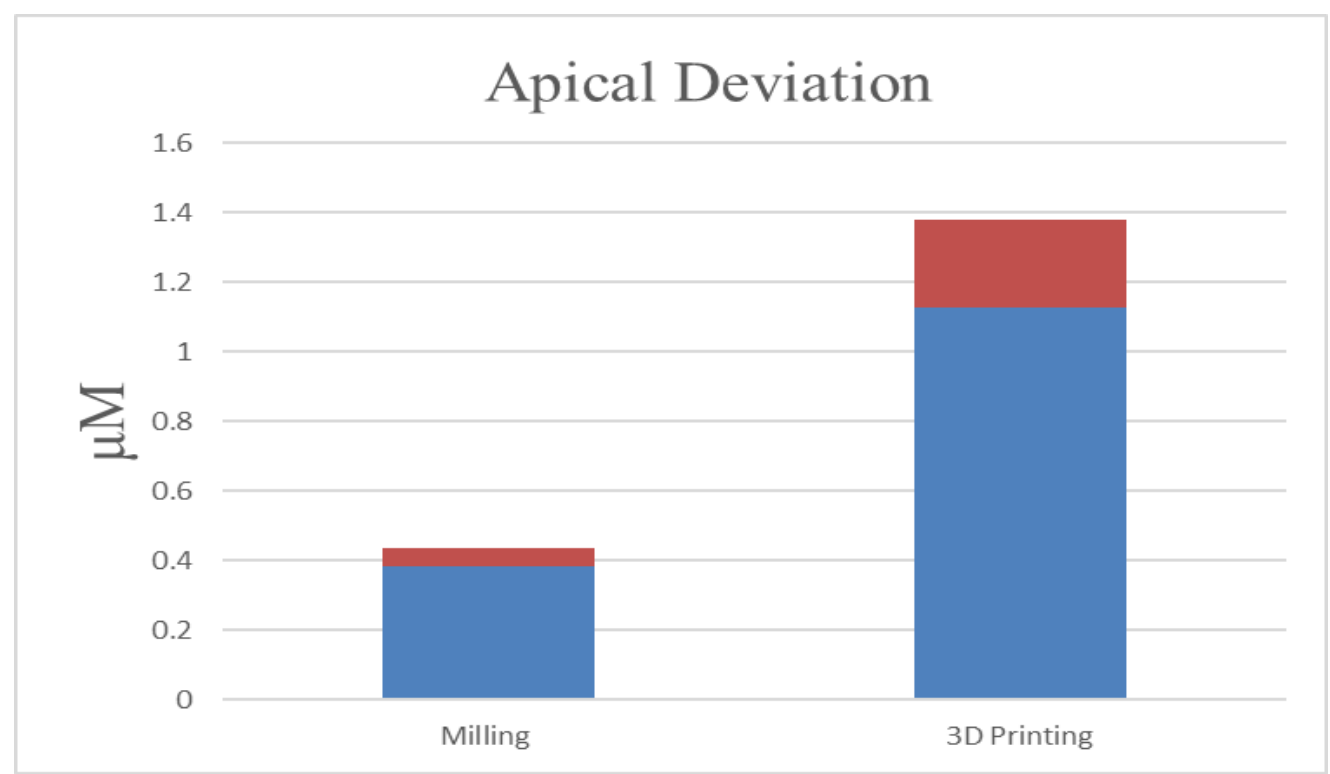

Figure 8: Bar chart representing mean and standard deviation values for apical deviation in milling and 3D printing surgical guides 


\section{DISCUSSION:}

Computer-assisted implant planning and subsequent template-guided implant placement must be highly accurate for optimal preoperative diagnostics and planning and, consequently, for developing a predictable procedure for implantation and prosthetic rehabilitation ${ }^{5}$.

The results of this study revealed statistically significant difference between the virtual implants planning and the actual implants in all aspects with higher values showed in group II (3D printing). In this study the significant difference of implants position may be due to some instability of surgical guides during surgery that may result in implants deviation, misplacement of the radiographic templates during scanning. Moreover, the technique used was partially limiting procedure as the implant insertion was made free hand that may result in some deviation, another possible source of variation is deformation of the surgical guide during prototyping.

On comparing the accuracy of implant positions of the two studied groups, the results revealed that both groups had higher apical deviation values than coronal deviation values. This finding may be explained by a study done by D'Haeseet al., (2012)6 which revealed the fact that implant guidance is most optimal in the coronal part of the prepared osteotomies because of the limited effect of the angular deviation at the entry point, which increases at a larger distance $^{6}$

This differences may be due to the following causes as using the same implant system; implant installation was carried-out by one operator, to exclude human variations in experience. Moreover, the scanning method was made with the aid of the same machine of CBCT used with all cases. Because the errors were cumulative, all the steps of the protocol may have contributed to the deviation.

Template-guided surgery concept involves many processes that result in deviations between the planned and the clinically placed implant positions. The overall accuracy of the implant placement is the sum of all errors that arise during the whole treatment procedure. Although it is difficult to detect deviations that possibly occur in each step, it is essential for clinicians to learn to what extent the deviations occur between the virtually planned implant positions and positions of clinically placed implants, in order to avoid anatomical risks as well as for the final prosthetic reconstruction. The accuracy is also a great matter of concern, especially in the case of immediate delivery of a prefabricated prosthesis ${ }^{7}$.

Inaccuracies of $3 \mathrm{D}$ printing compared to milling is attributed the need for supporting structure. While for the milling protocol of PMMA was very sensitive due to the excessive hardness of the material that in reverse increased the pressure of cutting that might lead to thermal stresses and distortion of the material. Also cutting conditions might cause excessive vibration that 
could exert more thermal and mechanical stresses on the work piece especially in areas with thin thickness during the procedure ${ }^{8}$

Results of this study also goes with other previous studies that reported deformation during SLA prototyping 9,10

It is worth noting that the length, angle, and proper position of an implant play critical roles in the placement procedure. The results of the present study found that the coronal, apical and angular deviations between the planned and actual implant position were less than the wellknown standard "safety zone" of $2 \mathrm{~mm}$ away from vital structures, with less deviations showed in CAD/CAM group ${ }^{11,12}$

This study showed that angular, coronal, and apical deviation are accurate enough to avoid the damage of major anatomical structure during the procedure.

In a study done to evaluate the accuracy of a 3D printing surgical guide determined by CBCT and model analysis, it was found that the deviation measured by CBCT is similar to that of other studies but angle deviation is somewhat higher. The reason for this is that the previous studies used surgical kit and implant fixture of the subsidiary company that makes surgical guide, while universal surgical guide kit is used in this study. Also, compared the previous studies, the more rearmost molars are included in this study. Reference marker was not used when taking $\mathrm{CBCT}$, so higher error occurred during overlapping preoperative and postoperative $\mathrm{CBCT}^{13}$.

On the other hand, an in vitro study was done to compare the accuracy of implant placement using 3D printed and machine milled surgical guides and it was concluded that no significant differences were found between both groups for any of the measurements 14 .

The present use of CAD/CAM processed surgical guides has provided a high degree of simplicity in morphologic diagnosis, determining the surgical procedure and establishing the subsequent prognosis. Moreover, CAD/ CAM technology has facilitated flapless surgeries by improvising on pre-surgical planning. They have also facilitated restoration-driven surgeries by integrating the restorative determinants into the surgical planning ${ }^{15}$.

The Stereolithography surgical guides derived from CT scan planning data were found to be highly accurate and easy to use in either bone-supported, tooth supported, or mucosa-supported configuration to minimize the possibility of postoperative peri-implant tissue loss and to overcome the challenge of soft-tissue management during or after surgery ${ }^{16,17,18}$

The clinical importance of these results may be relevant in such situations when multiple parallel but distant implants are placed, and where the degree of accuracy is critical for prosthetic restoration. Re-angulation or replacement of removable wearing parts could be reduced by the use of more accurate surgical implant placement ${ }^{19}$. 


\section{CONCLUSION}

Within the limitations of this study it may concluded that: The use of 3D printing and CAD/CAM milling surgical guides for flapless dental implant placement may result in a deviation of implants between virtual \& actual implants positions. Differences in Implants' deviations in the two studied groups were statistically significant in all deviations. The CAD/CAM group had superior results than 3D printing group.

\section{REFERENCE}

1. Kola MZ, Shah AH, Khalil HS, Rabah AM, Harby NMH, Sabra SA, et al. Surgical templates for dental implant positioning; current knowledge and clinical perspectives. Niger J Surg Off Publ Niger Surg Res Soc . 2015;21(1):1-5.

2. D’Souza KM, Aras MA. Types of implant surgical guides in dentistry: a review. J Oral Implantol, 2012;38(5):643-52.

3. Pal US, Chand P, Dhiman NK, Singh RK, Kumar V. Role of surgical stents in determining the position of implants. Natl J Maxillofac Surg [Internet]. 2010;1(1):203.

4. Lee WC, Huang $\mathrm{CH}$, Chung SC, Wei CC. An efficient and accurate approach for fabricating dental implant surgical guides. Biomed Mater Eng. 2014;24(6):2689-95.

5. Marchack CB. An immediately loaded CAD/CAM-guided definitive prosthesis: A clinical report. J Prosthet Dent 2005;93:8-12.

6. D'Haese, J., et al., A prospective study on the accuracy of mucosally supported stereolithographic surgical guides in fully edentulous maxillae. Clin Implant Dent Relat Res, 2012. 14(2): p. 293-303.

7. Vercruyssen M, Laleman I, Jacobs R, Quirynen M. Computer-supported implant planning and guided surgery: a narrative review. Clin. Oral Implants Res. 2015; 26:6976.

8. Abduo J, Lyons K, Bennamoun M. Trends in ComputerAided Manufacturing in Prosthodontics: A Review of the Available Streams. Int. J. Dent. 2014; 2014:e783948.

9. Silva, D.N., et al., Dimensional error in selective laser sintering and 3D-printing of models for craniomaxillary anatomy reconstruction. J Craniomaxillofac Surg, 2008. 36(8):443-9.

10. Stumpel, L.J., Deformation of stereolithographically produced surgical guides: an observational case series report. Clin Implant Dent Relat Res, 2012. 14(3): p. 442-453.

11. Valente F, Schiroli G, Sbrenna A. Accuracy of computer-aided oral implant surgery: a clinical and radiographic study. Int. J. Oral Maxillofac. Implants 2009; 24:234-42. 
12. -Worthington P. Injury to the inferior alveolar nerve during implant placement: a formula for protection of the patient and clinician. Int. J. Oral Maxillofac. Implants 2004;19:731-4.

13. Boyoung M, Park T, Chun I and Yun K. The accuracy of a 3D printing surgical guide determined by CBCT and model analysis. J Adv Prosthodont 2018;10:279-285.

14. -Ahmed MF, Ahmed M. AbdelHamid AM and AlAbbasy FH. Accuracy of Implant Placement Using Two Different Types of CAD/CAM Surgical Guides (An In vitro Study). Alex Dent J. 2019;44 : 28-33

15. -D’souza KM, Aras MA. Applications of computer-aided design/computer assisted manufacturing technology in dental implant planning. J Dent Implants. 2012; 2(1): 37 41

16. -Ozan O, Turkyilmaz I, Ersoy AE, McGlumphy EA, Rosenstiel SF. Clinical accuracy of 3 different types of computed tomography derived stereolithographic surgical guides in implant placement. J Oral Maxillofac Surg. 2009; 67:394-401.

17. Arisan V, Karabuda ZC, Özdemir T. Accuracy of two stereolithographic guide systems for computer-aided implant placement: a computed tomography-based clinical comparative study. J Periodontol. 2010;81:43-51

18. Rocci A, Martignoni M, Gottlow J. Immediate loading in the maxilla using flapless surgery, implants placed in predetermined positions, and prefabricated provisional restorations: a retrospective 3-year clinical study. Clin Implant Dent Relat Res. 2003; 5:29-36.

19. Nokar S, Moslehifard E, Bahman T, Bayanzadeh M, Nasirpouri F and Nokar A. Accuracy of Implant Placement Using a CAD/CAM Surgical Guide: An In Vitro Study Int J Oral Maxillofac Implants 2011;26:520-526.

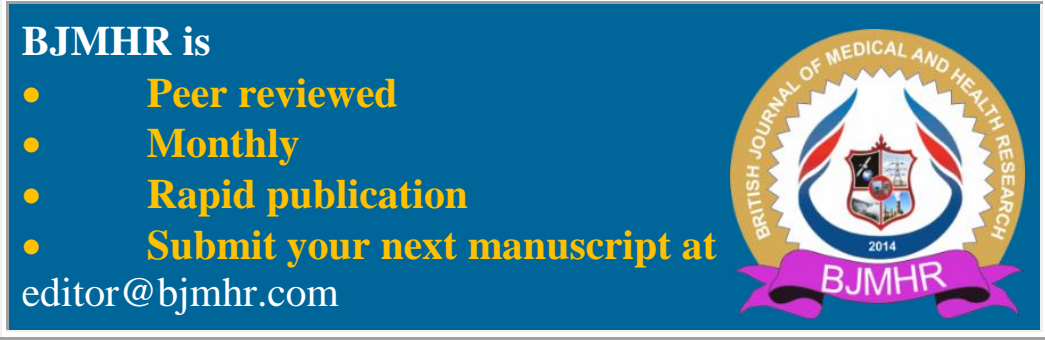

